\title{
Adaptive Filtering using Channel Representations
}

\author{
Michael Felsberg
}

\section{Linköping University Post Print}

N.B.: When citing this work, cite the original article.

Original Publication:

Michael Felsberg, Adaptive Filtering using Channel Representations, 2012, chapter in Mathematical Methods for Signal and Image Analysis and Representation, eds Luc Florack, Remco Duits, Geurt Jongbloe, Marie-Colette van Lieshout, Laurie Davies, pp 31-48.

http://dx.doi.org/10.1007/978-1-4471-2353-8_2

Copyright: Springer

Postprint available at: Linköping University Electronic Press

http://urn.kb.se/resolve?urn=urn:nbn:se:liu:diva-74735 


\title{
Adaptive Filtering using Channel Representations *
}

\author{
Michael Felsberg
}

Abstract This review article gives an overview on adaptive filtering methods based on channel representations. The framework of channel representations and its relation to density estimation is introduced. The fast and accurate scheme of virtual shift decoding is introduced and applied in several variants of channel smoothing:

- channel smoothing with alpha-synthesis for improving stability of edge-enhancing filtering

- orientation adaptive channel smoothing with applications to coherence-enhancing filtering

- channel smoothing using graph-cuts for improving filtering results at corners

- channel-coded feature maps (CCFMs) which lead to a significant speed-up of channel averaging

- CCFM-based smoothing based on optimal parameters derived from a novel uncertainty relation

For each method, an algorithmic description and some examples of results are provided, together with discussions and references of the original papers. Cross connections to other articles in this volume are given where appropriate.

\section{Introduction}

Adaptive filtering is an important field of image processing that has been considered by many researchers during the past three decades. Many different variants of

Computer Vision Laboratory, Linköping University, SE-58183 Linköping, Sweden e-mail: michael.felsberg@liu.se

* The research leading to these results has received funding from the European Community's Seventh Framework Programme (FP7/2007-2013) under grant agreements $n^{\circ} 215078$ (DIPLECS) and 247947 (GARNICS) as well as the VR project 2009-4282. 
adaptivity have been discussed in the literature, but the most relevant instances are probably orientation and scale.

The concept of scale was first introduced systematically in terms of the concept of linear scale-space [30, 55, 37], establishing a 3D space of spatial coordinates and a scale coordinate. Often identified with Gaussian low-pass filtering, a rigorous analysis of underlying scale-space axioms [53] has led to the discovery of the Poisson scale-space [18] and more general $\alpha$ scale-spaces [8].

In practice, discrete scale-spaces are mostly sub-sampled with increasing scale parameter, leading to the concept of scale-pyramids [25,5], multi-scale analysis and wavelet theory $[42,6,24]$. While pyramids and wavelets speedup the computation of linear operators and transforms, non-linear scale-space methods are widely used, e.g. for image enhancement. Non-linear scale-space is based on a non-stationary or anisotropic diffusivity function [44, 52, 27, 19].

More recently, non-linear methods have been introduced which are less directly connected to linear scale-space space and diffusion, but allow for faster processing and partially superior results $[46,14]$. The former method is based on wavelets, whereas the latter one is based on the channel representation [26] and is called channel smoothing. Combining the channel representation with a systematic decimation of spatial resolution, similar to the pyramid approach, has been applied in blob-detection [21] and in channel-coded feature maps (CCFM) [31, 34], a density representation in spatio-featural domain, see also [16].

Non-linear filtering based on anisotropic diffusivity is closely related to orientation adaptive filtering [12]. Orientation adaptive filtering, often referred to steerable filters [23], goes actually back on the work [36]. In more recent work, it has been extended to orientation selective channel smoothing [15] and orientation scores and their processing [7].

This review paper focusses on the channel-based variants of adaptive filtering and it is structured as follows. The framework of channel representations and its relation to density estimation is introduced in Section 2. The fast and accurate scheme of virtual shift decoding is introduced in Section 3. Sections 4-8 summarize several variants of channel smoothing:

4. channel smoothing with alpha-synthesis for improving stability of edge-enhancing filtering

5. orientation adaptive channel smoothing with applications to coherence-enhancing filtering

6. channel smoothing using graph-cuts for improving filtering results at corners

7. channel-coded feature maps (CCFMs) which lead to a significant speed-up of channel averaging

8. CCFM-based smoothing based on optimal parameters derived from a novel uncertainty relation

For each method, an algorithmic description and some examples of results are provided, together with discussions of the original papers. The paper is concluded with some general comments and an extensive list of references. 


\section{The channel representation}

Channel coding, also called population coding [56, 47], is a biologically inspired data representation, where features are represented by weights assigned to ranges of feature values [29, 26], see Fig. 1. Similar feature representations can also be found in the visual cortex of the human brain, e.g. in the cortical columns.

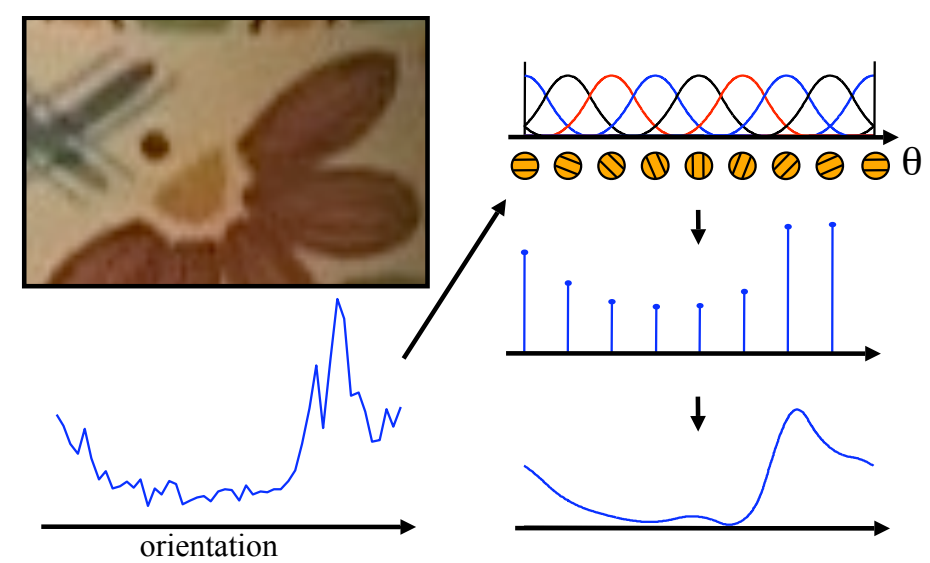

Fig. 1 Orientation distribution is encoded into channels, resulting in a (low-pass filtered) reconstruction using maximum entropy [32], see also [40]. Figure courtesy Erik Jonsson.

The closer the current feature value $f$ to the respective feature interval center $n$, the higher the channel weight $c_{n}$ :

$$
c_{n}(f)=k(f-n) \quad n \in \mathbb{N},
$$

where $k(\cdot)$ is a suitable kernel function and where $f$ has been scaled such that it has a suitable range (note that we chose to place the channel centers at integers). By introducing $z$ as a continuous feature coordinate, $k_{n}(z)=k(z-n)$, and $\boldsymbol{\delta}_{f}(z)=\boldsymbol{\delta}(z-$ $f$ ) denoting the Dirac-delta at $f$, the encoding can be written as a scalar product

$$
c_{n}(f)=\left\langle\delta_{f} \mid k_{n}\right\rangle=\int \delta_{f}(z) k_{n}(z) d z
$$

or as a sampled correlation in the feature-domain:

$$
c_{n}=\left(\delta_{f} \star k\right)(n)=\left.\int \delta_{f}\left(z^{\prime}\right) k\left(z^{\prime}-z\right) d z^{\prime}\right|_{z=n} .
$$

From the weights of all channels the feature value can be decoded unambiguously by finding the mode, where the decoding depends on the kernel function. In what follows, we have been using quadratic B-splines: 


$$
B_{2}(z)= \begin{cases}(z+3 / 2)^{2} / 2 & -3 / 2<z \leq-1 / 2 \\ 3 / 4-z^{2} & -1 / 2<z \leq 1 / 2 \\ (z-3 / 2)^{2} / 2 & 1 / 2<z<3 / 2 \\ 0 & \text { otherwise }\end{cases}
$$

This results in the encoding scheme Alg. 1, where $N$ denotes the number of channels.

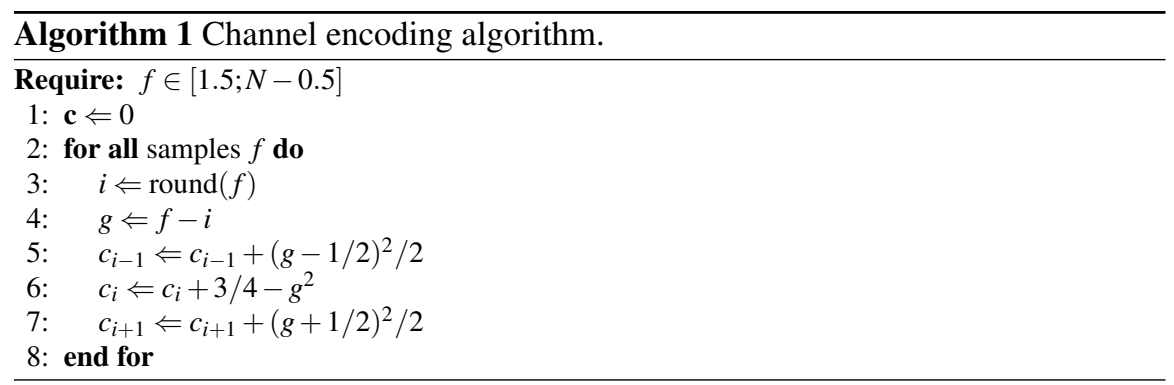

The features can be scalar valued or vector valued, e.g. greyscales, color vectors, or orientations. In the case of scalar features the decoding from quadratic B-splines has been considered in detail in [14], see next section. For the case of non-interfering channel weights, a simplified scheme based on the quotient of linear combinations can be used:

$$
M_{n}=c_{n-1}+c_{n}+c_{n+1} \quad n_{0}=\arg \max M_{n} \quad \hat{f}=\frac{c_{n_{0}+1}-c_{n_{0}-1}}{M_{n_{0}}}+n_{0}
$$

where $\hat{f}$ is our estimate of the feature $f$ that had been encoded in $c_{n}$.

\section{Virtual Shift Decoding}

The decoding scheme (5) is not sufficient in all applications due to its quantization effects, i.e., the decoding result is biased toward the channel centers. If avoiding quantization effects in the decoding is essential, the virtual shift decoding [14] can be applied. For its derivation the reader should refer to the original paper. The algorithm works as follows. Let $h=2 \sqrt{2}-3$. In a first processing step, the channels are forward and backward filtered according to

$$
\begin{aligned}
c_{n}^{+} & =c_{n}+h c_{n-1}^{+}, \quad(n=2, \ldots, N) \\
c_{n}^{-} & =h\left(c_{n+1}^{-}-c_{n}^{+}\right), \quad(n=N-1, \ldots, 1) \\
c_{n}^{\prime} & =8 c_{n}^{-} .
\end{aligned}
$$


For finite domains, the boundary conditions are given as

$$
\begin{aligned}
c_{1}^{+} & =c_{1} \\
c_{N}^{-} & =\frac{h}{h^{2}-1} c_{N}^{+} .
\end{aligned}
$$

For periodic domains, a DFT-based method should be applied instead of (6-10)

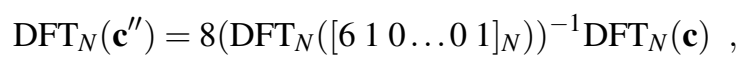

where $\mathrm{DFT} T_{N}$ denotes the $N$-point DFT along the channel index. The new channel vector $\mathbf{c}^{\prime \prime}$ must be extended at both ends according to

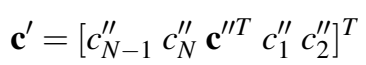

in order to process it further as if it was non-periodic. Sampling the continuous function

$$
p(f)=\sum_{n=1}^{N} c_{n}^{\prime} B_{2}(f-n)
$$

results in the original channel vector $\mathbf{c}$ again, i.e., $p(f)$ is a proper interpolation of the channel vector.

In order to extract the modes $f_{n}$ at channels $n$, define $\beta_{n}=f_{n}-n$ and solve

$$
\begin{aligned}
0 & =\lambda \beta_{n}^{2}+\mu \beta_{n}+v \quad \text { with } \\
\lambda & =\left(c_{n_{0}-2}^{\prime}-2 c_{n_{0}-1}^{\prime}+2 c_{n_{0}+1}^{\prime}-c_{n_{0}+2}^{\prime}\right) / 2 \\
\mu & =\left(-c_{n_{0}-2}^{\prime}+2 c_{n_{0}}^{\prime}-c_{n_{0}+2}^{\prime}\right) / 2 \\
\nu & =\left(c_{n_{0}-2}^{\prime}+6 c_{n_{0}-1}^{\prime}-6 c_{n_{0}+1}^{\prime}-c_{n_{0}+2}^{\prime}\right) / 8
\end{aligned}
$$

such that the minimum of the error corresponds to

$$
\beta_{n}=\frac{-\mu / 2+\sqrt{\mu^{2} / 4-v \lambda}}{\lambda}
$$

Solutions where $\left|\beta_{n}\right|>1 / 2$ must be excluded, since they are located outside the unit interval around $n$. For valid solutions of $\beta_{n}$, the decoded mode is given by $f_{n}=n+\beta_{n}$. For periodic domains, the correct values are obtained after a modulo operation.

In order to extract the maximum likelihood estimate or to order the modes, the robust error needs to be known. It is given at channel $n$ as

$E(n)=\frac{23}{24}+\beta_{n} v+\beta_{n}^{2} \mu / 2+\beta_{n}^{3} \lambda / 3-\frac{c_{n_{0}-2}^{\prime}+24 c_{n_{0}-1}^{\prime}+46 c_{n_{0}}^{\prime}+24 c_{n_{0}-1}^{\prime}+c_{n_{0}-2}^{\prime}}{48}$.

The virtual shift decoding algorithm is summarized in Alg. 2. 


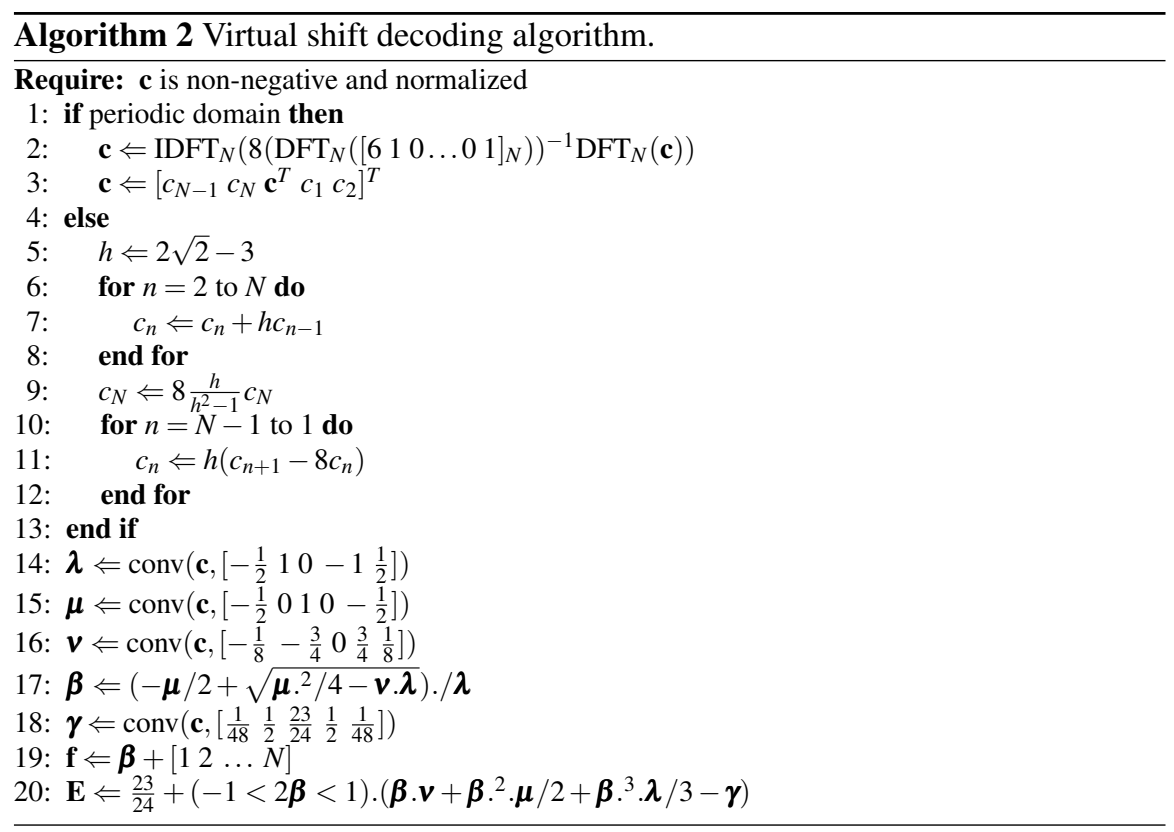

Channel representations obviously need more memory than directly storing features, but this investment pays off in several ways which we will show in the subsequent sections.

\section{Channel Smoothing}

The idea of channel smoothing is based on considering the feature $f$ in the encoding (1) as a stochastic variable. It has been shown in [14] that the distribution $p_{f}$ is approximated by $c_{n}$ in expectation sense (see also Fig. 2):

$$
E\left\{c_{n}(f)\right\}=\left(p_{f} \star k\right)(n)
$$

such that $\hat{f}$ becomes a maximum-likelihood estimate of $f$.

If we assume that $p_{f}$ is locally ergodic, we can estimate $\hat{f}$ from a local image region, which corresponds to a local averaging of the channel weights within a spatial neighborhood. The algorithm consisting of the three steps channel encoding, channel averaging, and channel decoding is called channel smoothing (see Alg. 3 and Fig. 3) and has been shown to be superior to many other robust smoothing methods $[14,50]$. Due to its structure, Alg. 3 is very well suited for parallel implementations. A nearly identical scheme has been proposed independently, but later, in [43]. 

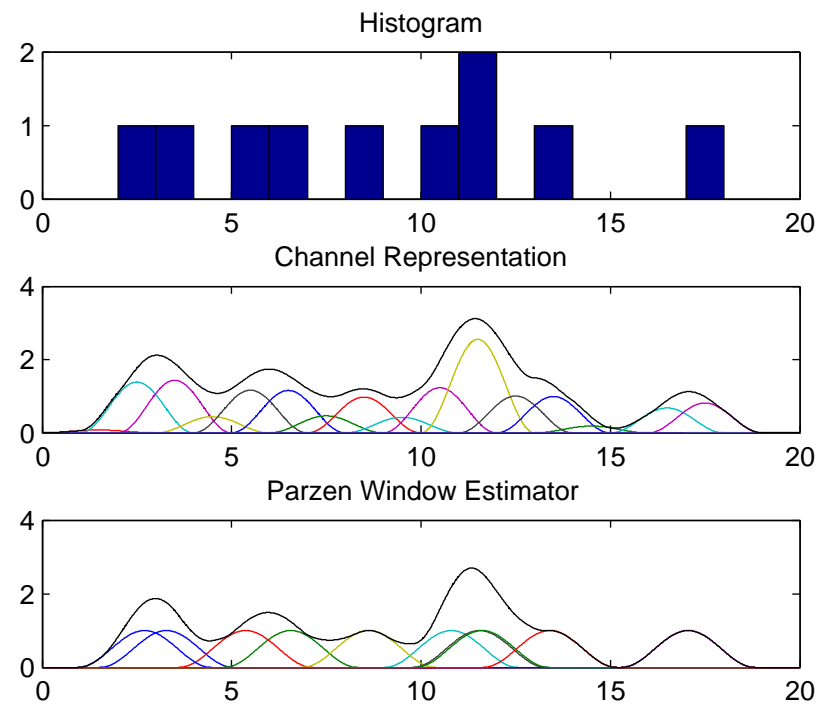

Fig. 2 Schematic comparison of density estimation using a histogram (top), a channel representation (middle), and a Parzen window estimator (bottom).

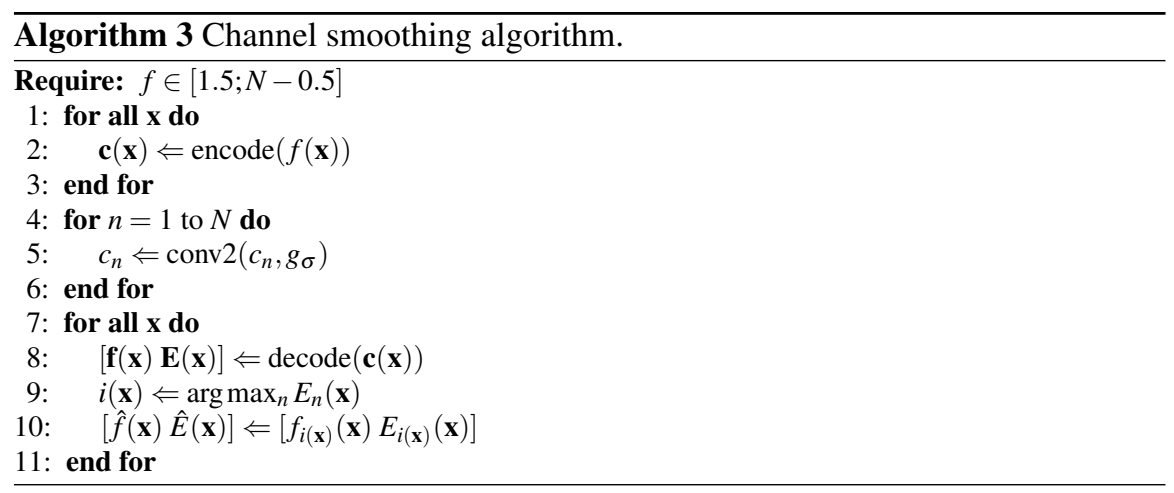

Due to the point-wise decoding in Alg. 3, the positioning of region boundaries might violate the sampling theorem, resulting in unstable edge-pixels. To avoid this effect, a modification to the channel decoding has been proposed in [20], called $\alpha$ synthesis, which creates smooth transitions between neighborhoods with different feature levels. Instead of extracting only the maximum in (5) or Alg. 3, line 9, all local decodings are combined, weighted by the respective robust errors

$$
\hat{f}=\frac{\sum_{n} f_{n}\left(\frac{23}{24}-E_{n}\right)^{\alpha}}{\sum_{n}\left(\frac{23}{24}-E_{n}\right)^{\alpha}} .
$$



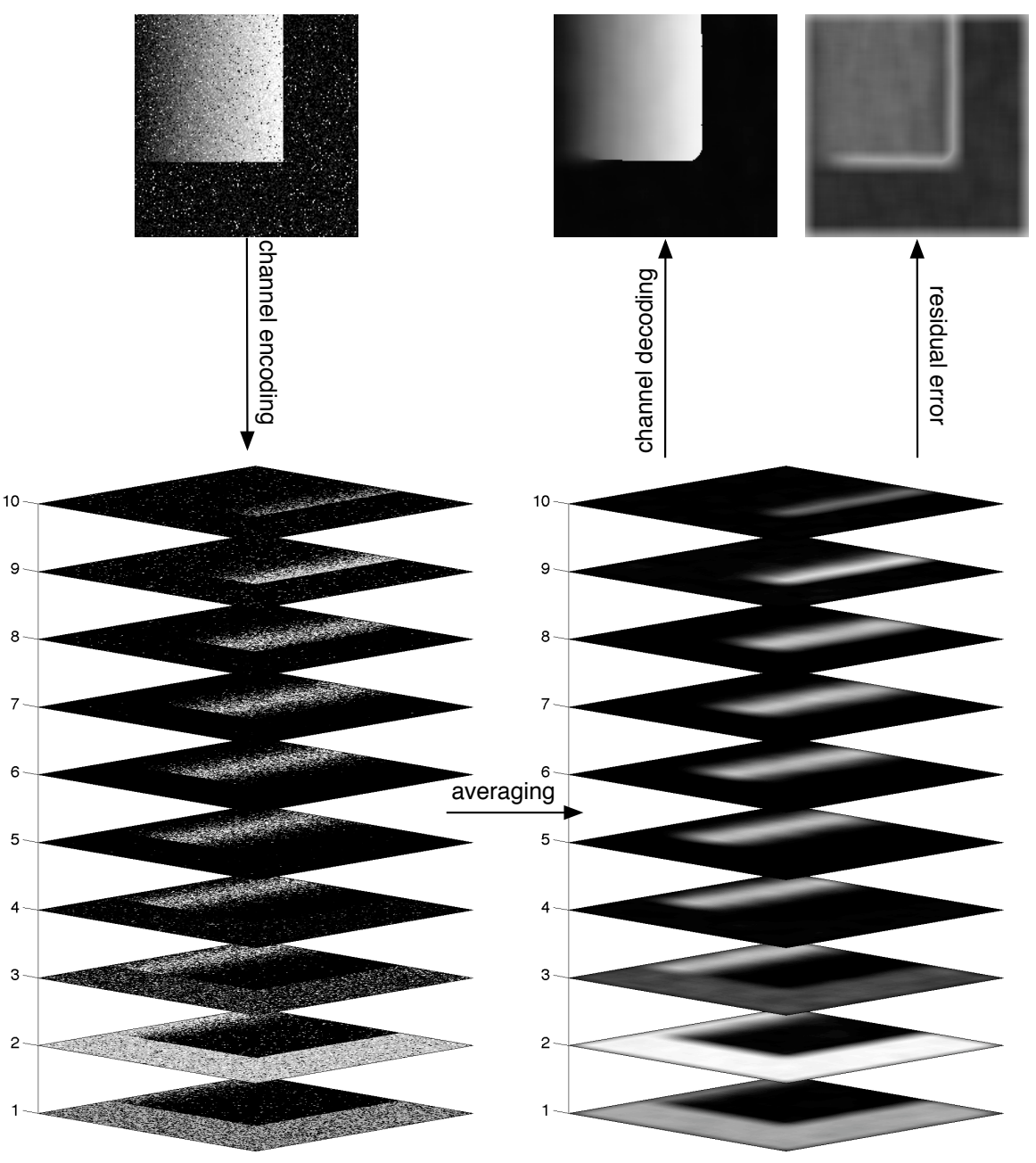

10

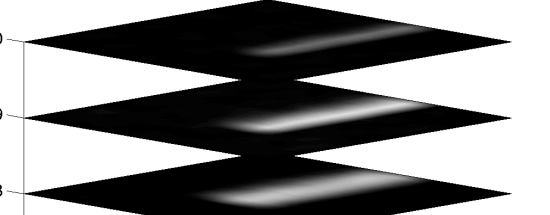

8
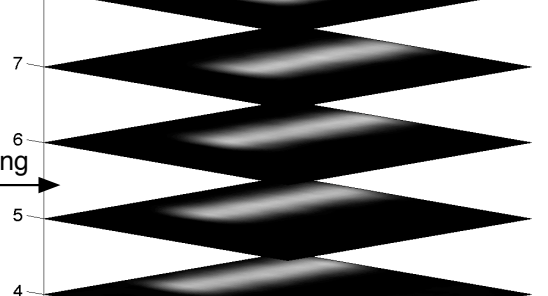

4

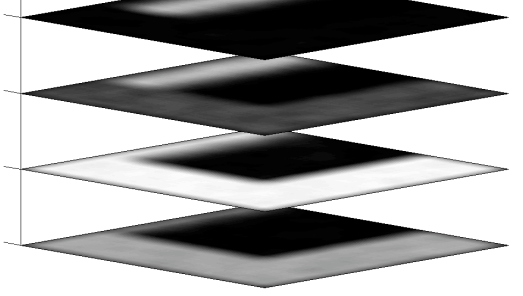

Fig. 3 The simple synthetic example (top left) smoothed by channel smoothing, using a gaussian filter with $\sigma=10$. No quantization effects are visible but note the rounding of the corner. On the top right: robust error $E$ of the decoding.

For the choice of $\alpha$ see [20]; we used $\alpha=2$ throughout this paper. This method avoids aliasing artefacts at edges, but it does not avoid the rounding of corners, see Fig. 4. 

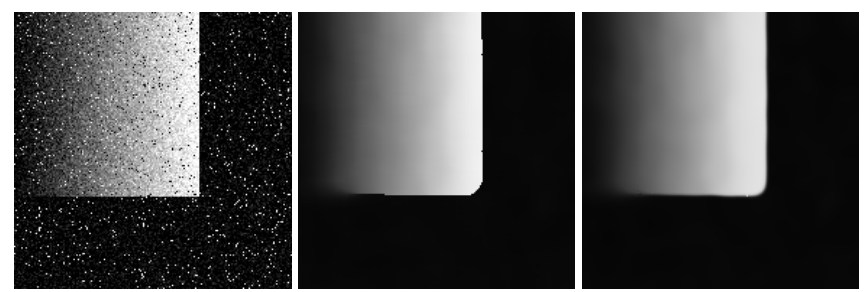

Fig. 4 From left to right: original noisy test image, result without alpha-synthesis (not the flipovers st the edges), and result with alpha-synthesis.

\section{Orientation Adaptive Channel Smoothing}

The contents of this section is based on the publication [15], but the work is also closely related to $[1,4]$, as well as [41].

Channel smoothing as introduced in the previous section preserves edges, similar to edge-enhancing diffusion. Close to an edge, the filter support of a comparable linear filter is a semi-disc lying entirely on one side of the edge, thus giving a stable response everywhere except for points lying exactly on the edge. The latter problem has been solved by $\alpha$-synthesis. For elongated, line-like structures, for instance in images of finger prints, the ordinary channel smoothing becomes however instable, as the majority of points are edge points. As a result, the resulting image suffers from 'flip-over' effects and contours become fragmented or displaced.

This problem is avoided if the channel smoothing is only performed along a 1D subspace, given by the local signal orientation $\theta_{f}$, similar to the principle of adaptive filters [36] or coherence enhancing diffusion [54]. Hence, the channel smoothing operation must depend on the local signal orientation, which is itself represented using channels. The orientation information is typically of much slower variation than the intensity information and thus ordinary channel smoothing gives good results [14].

The smoothed orientation channels and the greyscale channels are then combined in an outer product, see Fig. 5. The channel matrix encodes explicitly which greyscale is present at which orientation. Hence, it is straightforward to adapt the smoothing kernel to the local orientation: we simply use differently oriented anisotropic smoothing kernel $g_{m}$ for each orientation channel.

This smoothed, extended channel representation is then decoded in three steps using the standard decoding: Decode the orientation channel for each greyscale, use the obtained error estimates to build a new greyscale channel vector, and decode the new channel vector. The complete algorithm is summarized in Alg. 4.

The ratio behind the sketched method is as follows. For oriented structures the channel matrix has a clear 2D maximum. Generating the greyscale channel vector in line 16 yields a vector with a maximum at the appropriate greyscale. The effective filter kernel is dominated by the anisotropic kernel corresponding to the strongest orientation channel, i.e., the structure is smoothed along its orientation and maintained perpendicular to its orientation, see Fig. 6. 


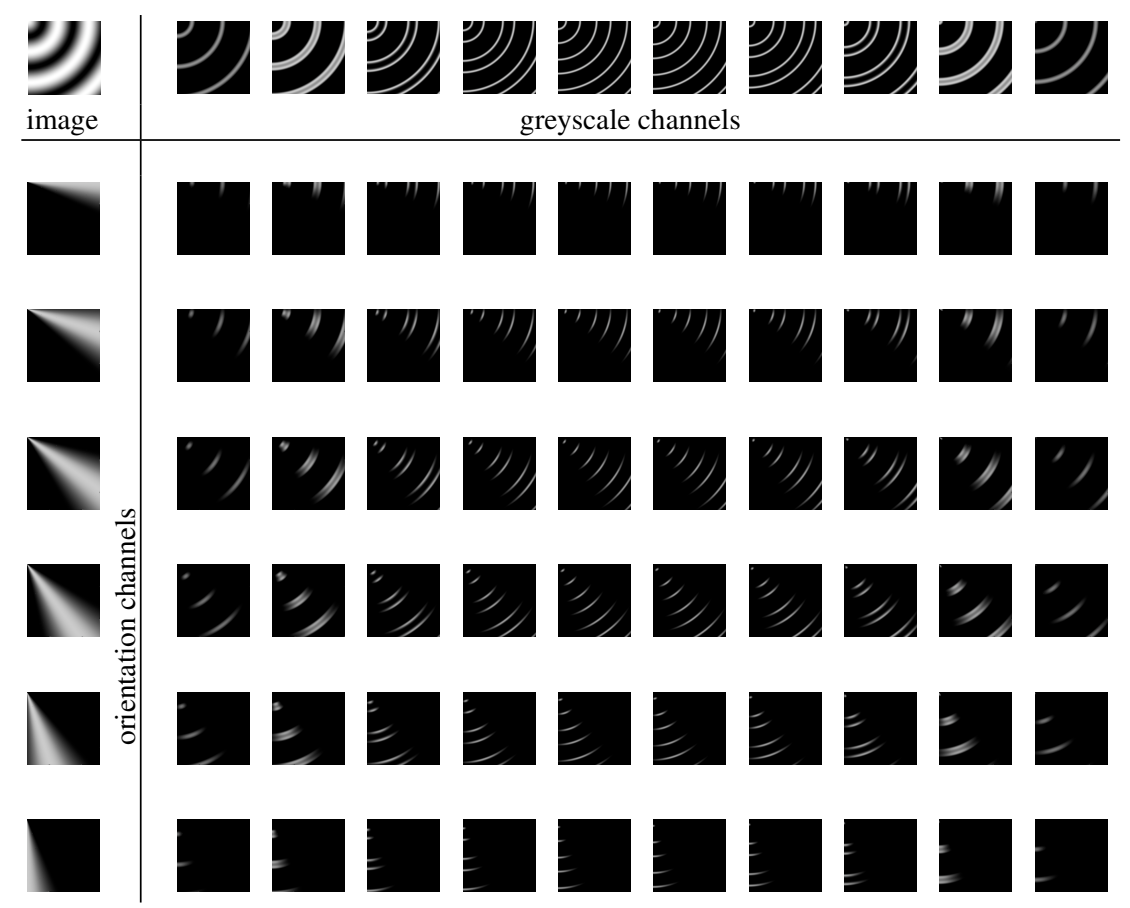

Fig. 5 Channel matrix using ten greyscale channels and six orientation channels.

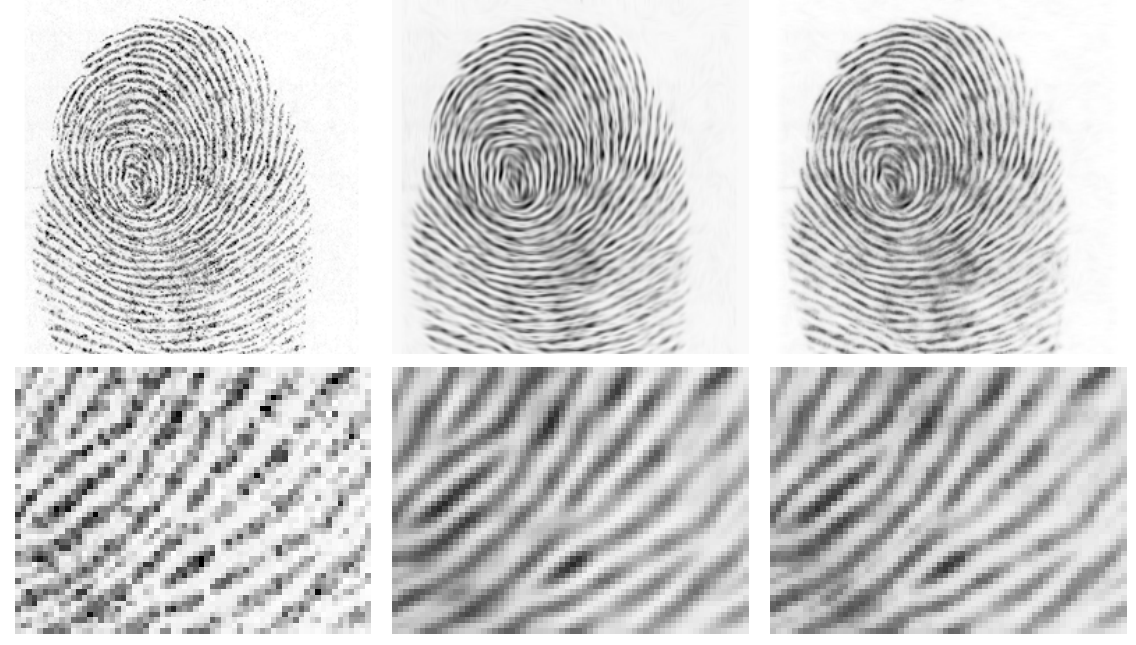

Fig. 6 Fingerprint experiment from [15]. From left to right: original images, results from coherence enhancing diffusion [2], and results from channel smoothing. Top: fingerprint at 300dpi, bottom: zoomed detail. For further details and parameters, refer to [15]. 


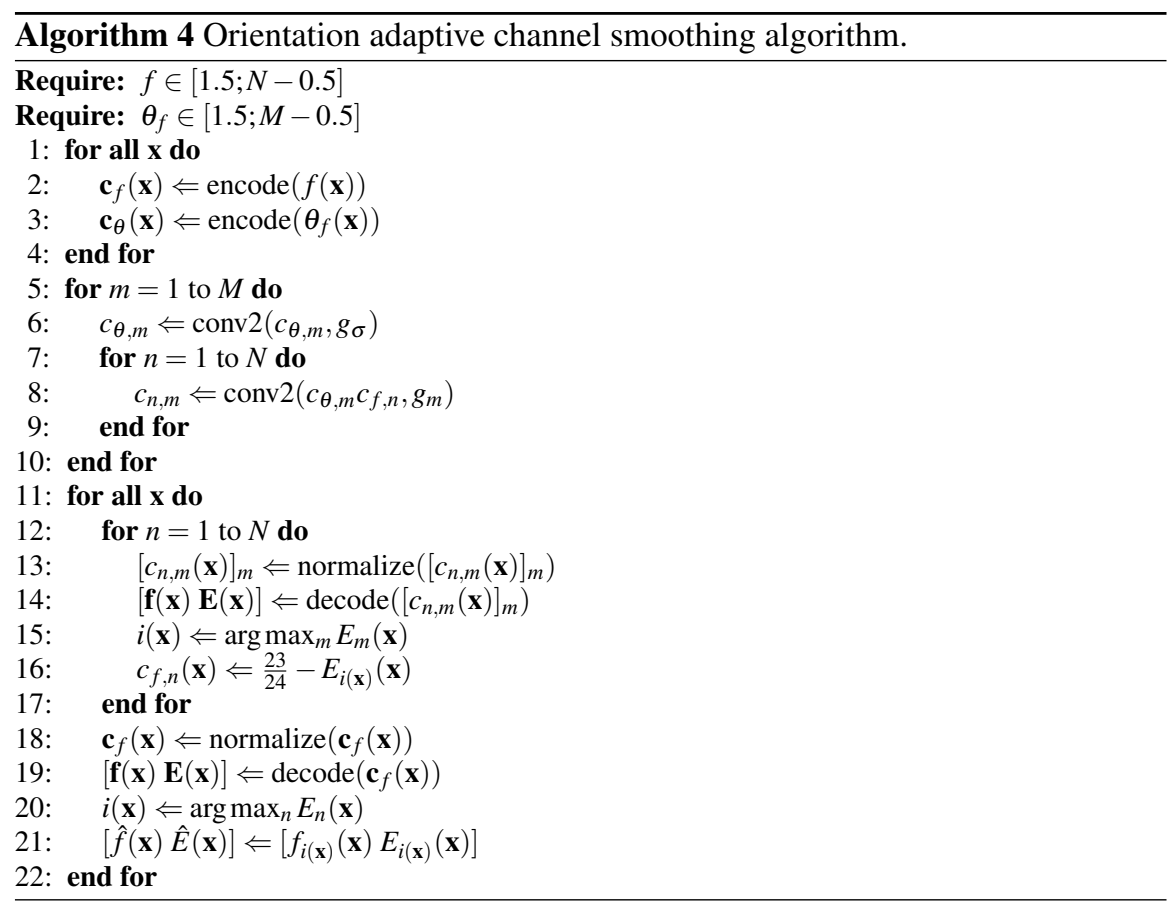

For unoriented structures, the orientation decoding is random, but the resulting greyscale channel will still be correct as the greyscales are identical for all orientations. The effective filter kernel is a combination of anisotropic kernels with random orientation, thus resulting in an isotropic kernel. Hence, the filter output corresponds to isotropic smoothing.

A further aspect of adaptive filtering is the choice of the smoothing kernels, depending on the noise level [10] (for related work on noise level estimation, see also [17, 48]) and the noise distribution, e.g., multiplicative noise [49]. The selection of filter kernels is however out of the scope of this review and the interested reader is referred to the original publications.

\section{Channel Smoothing without Corner Rounding}

The method described in this section is based on the publication [11], which proposes to avoid rounding of corners by restricting the smoothing to a generic domain where the respective channels are active. This means in practice: channel values should be averaged on bounded domains. Before looking into the issue of determining the active region of a channel, we have to modify the averaging step in channel smoothing in order to apply it to a bounded domain. Filtering of (uncertain) data $f$ from a bounded domain is well modeled in terms of normalized convolution [35] of 
0th order (normalized averaging):

$$
\hat{f}=\frac{(a *(b f))}{(a * b)}
$$

where $a$ denotes the applicability function, i.e., a suitable averaging kernel (typically a Gaussian function), $b$ is the certainty function, which describes the bounded domain $\Omega$ :

$$
b(\mathbf{x})=\left\{\begin{array}{ll}
1 & \mathbf{x} \in \Omega \\
0 & \mathbf{x} \notin \Omega
\end{array},\right.
$$

and $*$ denotes the convolution operation.

The co-domain of normalized convolution is however unbounded, and therefore, we cannot apply it directly to the case of channels $c_{n}$ that are active in a bounded region. To remain within the active region of each channel, we mask the result from normalized convolution to the same bounded domain of each channel by means of the certainty function $b_{n}$ :

$$
\hat{c}_{n}=b_{n} \frac{\left(a *\left(b_{n} c_{n}\right)\right)}{\left(a * b_{n}\right)} .
$$

What remains to be considered is the estimation of the active region, or equivalently, the certainty function for each channel. We have to find those regions where the image was sufficiently stationary in order to produce similar channel vectors $\mathbf{c}(\mathbf{x})$. Similar channel vectors have the same active components, where we classify the activity by a simple theshold $\theta$. As stationarity does not make sense without spatial context, we require the active region to be as connected as possible.

For each channel $n$ we formulate the following objective function:

$$
E\left(b_{n}\right)=\sum_{\mathbf{x}} b_{n}(\mathbf{x})\left(\boldsymbol{\theta}-c_{n}(\mathbf{x})\right)+\lambda \sum_{\{\mathbf{x}, \mathbf{y}\} \in \mathscr{N}}\left|b_{n}(\mathbf{x})-b_{n}(\mathbf{y})\right|,
$$

where we use the following parameters throughout the remainder of this paper:

- $\mathscr{N}$ is the four-neighborhood

- $\lambda=0.3$ is the penalty for discontinuities in $b_{n}$

- $\theta=\frac{1}{16}$ is the threshold for active channels.

All parameters were chosen according to [11] (as is the width of the Gaussian filter $\sigma=10$ and the number of channels $N=10$ ). The interested reader is referred to [10, 22] for estimation of the channel averaging filter and the number of channels. The threshold $\theta$ can be derived from classical decision theory (see e.g. [51], Chapt. 3) and depends also on the number of channels. The meta parameter $\lambda$ depends on the neighborhood structure and the signal statistics. It should be at least one third of the maximum channel value (minus $\theta$ ) to fill in one-pixel gaps in a contour with four-neighborhood. Too large values will remove structural details from the active region. For quadratic $B$-spline channels and $\theta=\frac{1}{16}$ this happens for $\lambda \geq \frac{11}{32}$.

A binary labelling problem as formulated in (24) (see also [28]) is efficiently solved by graph-cut algorithms [3]. Using graph-cut for determining the activation 
of channels, we obtain the graph-cut channel smoothing algorithm as given in Alg. 5 . The synthetic example from Fig. 3 shows that graph-cut channel smoothing does not suffer from the drawback of multi-label graph-cut (coarse quantization), nor does it suffer from rounding of corners as pure channel smoothing does, see Fig. 7. The computational complexity of the proposed method is somewhat higher than that of pure channel smoothing and it is dominated by the $N$ binary graph-cut computations.

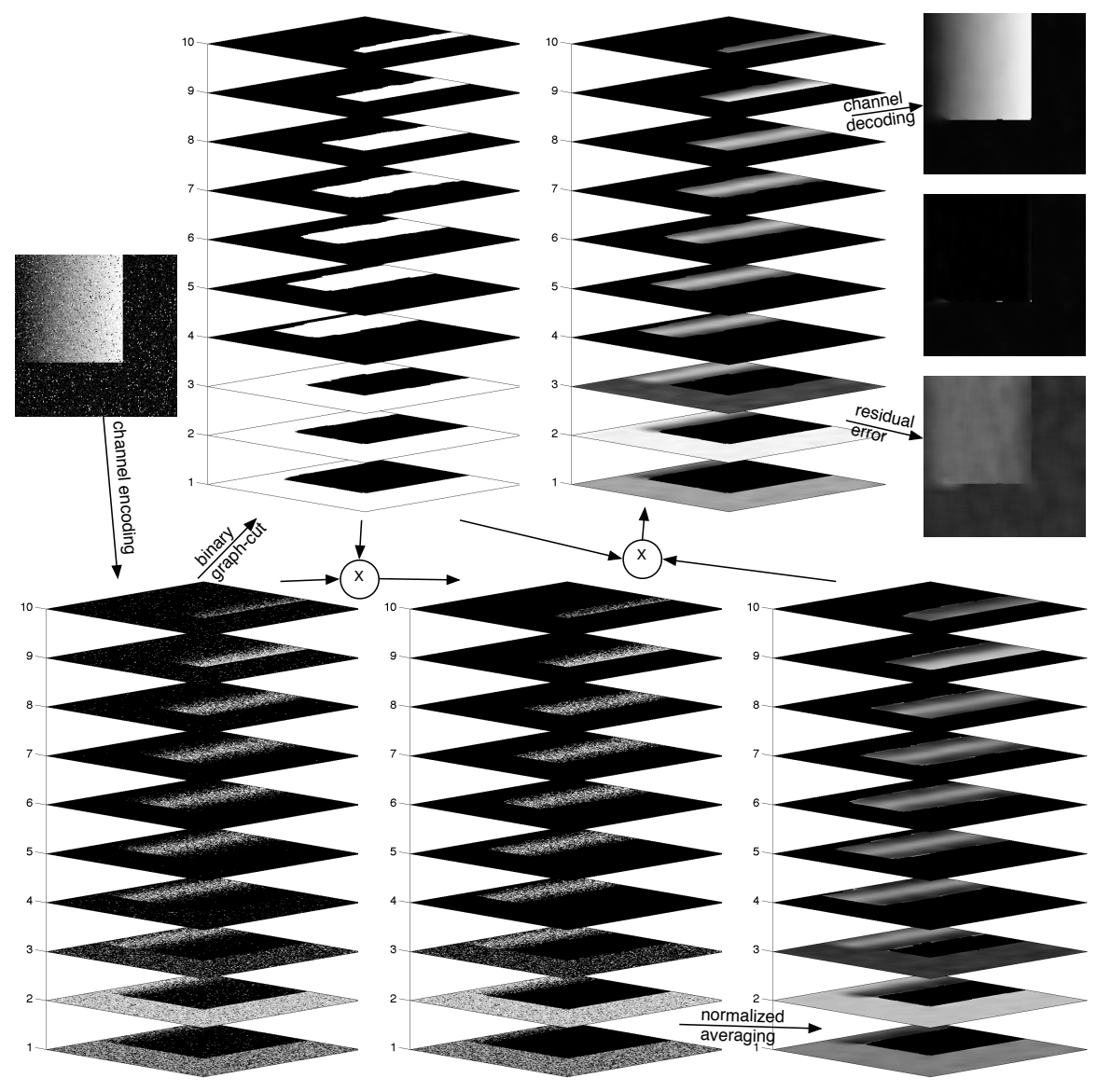

Fig. 7 Simple synthetic example from Fig. 3 smoothed by graph-cut channel smoothing, using a gaussian filter with $\sigma=10$. Top right: result from graph-cut channel smoothing. Below: actual absolute error compared to noise-free gradient image and reconstruction error. Note the absence of rounding of the corner. 


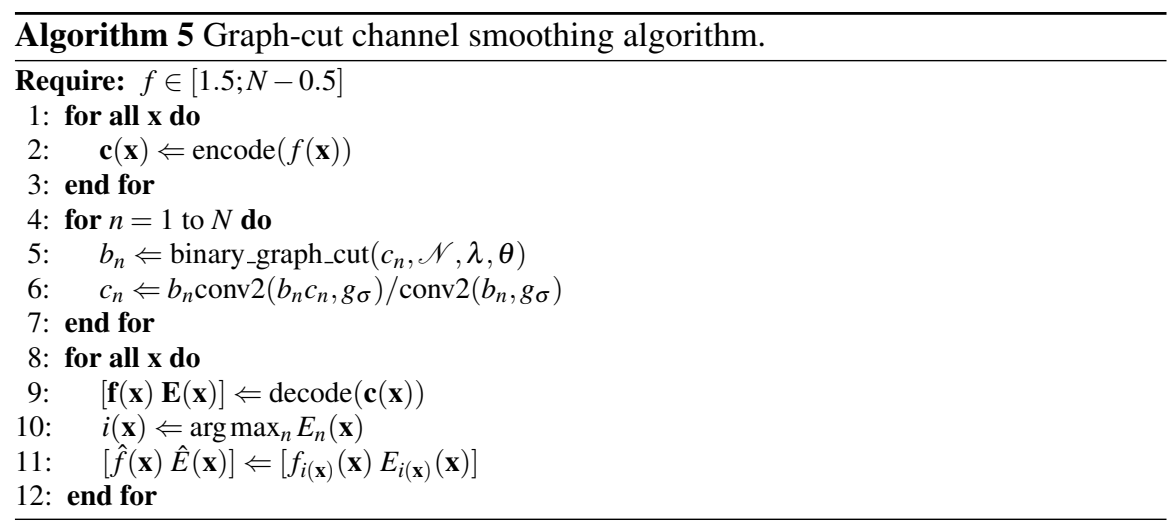

\section{Channel-Coded Feature Maps}

Channel-coded feature maps have been suggested in [33] and are related to [45] in a way that spatio-featural densities are estimated using channel representations.

One major drawback of channel smoothing is the extensive use of memory if many feature channels are required. A high density of channels is only reasonable if the spatial support is large, which implies that the individual feature channels are heavily low-pass filtered along the spatial dimension. Therefore, the feature channels have a lower band limit and can be subsampled in the spatial domain without losing information. If the three steps of channel encoding, channel averaging, and subsampling are integrated into a single step, channel-coded feature maps (CCFMs) are generated. The advantage of CCFMs is a much higher number of channels, e.g. by combining several features as in Fig. 8, without increasing the memory requirements significantly.

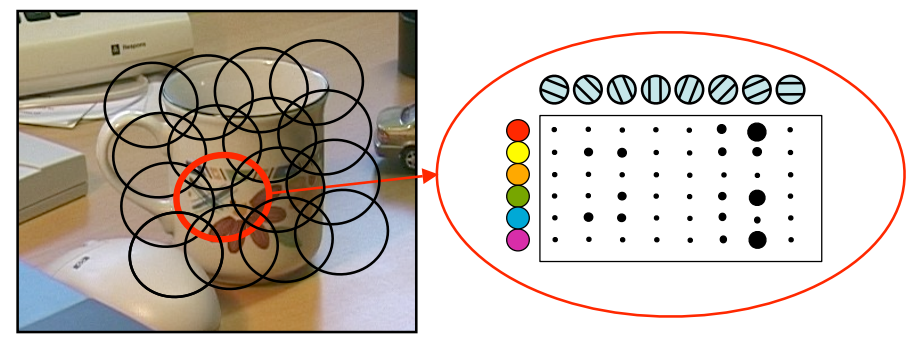

Fig. 8 Simultaneous encoding of orientation and color in a local image region. Figure taken from [31] courtesy Erik Jonsson.

The CCFM encoding of a single feature point can be written as (cf. (1)): 


$$
c_{l, m, n}(f(x, y), x, y)=k_{f}(f(x, y)-n) k_{x}(x-l) k_{y}(y-m),
$$

where $k_{f}, k_{x}, k_{y}$ are the 1D kernels in feature domain and spatial domain. Note that $x$ and $y$ are scaled such that they suit the integer spatial channel centers $l, m$. Similar to (1), the encoding (25) of a set of feature points can be written as a scalar product in $3 \mathrm{D}$ function space or as a $\mathrm{D}$ correlation, where we use

$$
\delta_{f}(x, y, z)=\delta(z-f(x, y))
$$

and $k_{f, n}(z)=k_{f}(z-n), k_{x, l}(x)=k_{x}(x-l), k_{y, m}(y)=k_{y}(y-m)$ :

$$
\begin{aligned}
c_{l, m, n}(f) & =\left\langle\delta_{f} \mid k_{f, n} k_{x, l} k_{y, m}\right\rangle=\iiint \delta_{f}(x, y, z) k_{f, n}(z) k_{x, l}(x) k_{y, m}(y) d z d y d x \\
& =\left(\delta_{f} \star\left(k_{f} k_{x} k_{y}\right)\right)(n, m, l) .
\end{aligned}
$$

The final formulation is the starting point of the CCFM scale-space, see next section. CCFMs can be computed very efficiently using monopieces [31,34], but we only present the straightforward algorithm here, see Alg. 6, where $\otimes$ denotes the outer (Kronecker) product. CCFMs and their derivatives can be used efficiently for robust visual tracking [33].

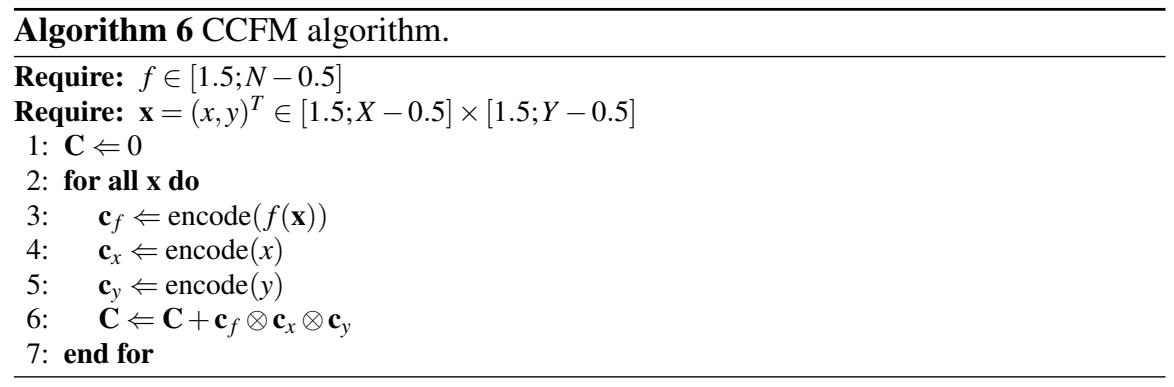

\section{CCFM Scale-Space}

This section summarizes some recent results [13] on the concept of CCFM scalespace and spatial-featural uncertainties.

The starting point is to embed the image $f(x, y)$ as a 3D surface according to (26). One might try to generate a 3D $\alpha$ scale-space [8] (Gaussian as a special case $\alpha=1$ and all $\alpha$-kernels are symmetric, i.e., correlation and convolution are the same):

$$
F_{s}(x, y, z)=\left(k_{s}^{(\alpha)} \star \delta_{f}\right)(x, y, z)
$$


However, the semi-group property of scale-space implies that all dimensions (spatial dimensions and the feature dimension) become increasingly blurred. Despite the fact that this implies a rapidly growing loss of information with increasing scale and a singular zero scale, this procedure is insensible from a statistical perspective and does not comply with the notion of scale selection [39, 9].

Since the latter argument is not straightforward, we explain our rationale in some more detail. From the requirement that the dimensionless derivative attains its maximum at a position proportional to the wavelength of the signal [39] (section 13.1), we conclude that the scale of a structure is proportional to its spatial scale (a trivial fact) and anti-proportional to its feature scale. The latter can be shown by looking at the Taylor expansion of a harmonic oscillation $A \sin (\omega x)$ in the origin: $A \omega x$. The steepness of a sinusoid $A \omega$ in the origin grows linearly with the amplitude and the frequency, i.e., it is antiproportional to the wavelength $\lambda=\frac{2 \pi}{\omega}$.

Alternatively, one can consider the energy of a harmonic oscillation. The energy is proportional to the square of the amplitude times the square of the frequency: $E \propto A^{2} \omega^{2} \propto \frac{A^{2}}{\lambda^{2}}$. That means, if we apply a 3D lowpass filter to the spatio-featural domain, the energy decays with a power of four. Hence, scale selection would favor the highest possible frequencies in nearly all cases. If we scale the amplitude anti-proportionally to the spatial domain, the change of energy is balanced and will reflect intrinsic properties of the signal.

This relation is formalized in terms of a spatio-featural uncertainty relation, which is derived based on the group structure of spatio-featural transformations. We choose a methodology which is based on the isotropic model used in [38], although restricted to the $1 \mathrm{D}$ case. The higher-dimensional case generalizes straightforwardly. The group that we consider contains the shearing group and the translation group given as

$$
\begin{aligned}
x^{\prime} & =x+t_{x} \\
f^{\prime} & =f+\tan (\phi) x+t_{f} .
\end{aligned}
$$

The shearing transformation corresponds to the rotation of a Euclidean space and is obtained since the $f$-coordinate is a null-vector [38], i.e., $f \cdot f=0$. The parameterization is chosen such that it reflects the fact that points move along the surface / curve with angle $\phi$ towards the ground plane. Using this definition we state the following

Theorem 1. Let the spatio-featural domain be described by the isotropic model. The uncertainty product in the spatio-featural domain has a lower bound

$$
\exists k>0: \quad(\Delta x)(\Delta f) \geq k
$$

and the lower bound is given as

$$
k=\frac{1}{2} \sigma_{f} \sigma_{x}
$$


where $\sigma_{f}^{2}$ is the variance of the feature domain marginal distribution and $\sigma_{x}^{2}$ is the variance of the spatial domain distribution.

The proof of this theorem is given in [13]. As a consequence of this theorem, optimal parameters for CCFM computation are derived. As an experimental validation, images have been reconstructed from these CCFMs showing that a good perceptual quality is maintained for a wide range of channels, see Fig. 9. The CCFM-smoothing algorithm is summarized in Alg. 7, where the numbers of channels $X, Y$, and $N$ are the optimized parameters mentioned before and the interpolation in line 3 generates a new channel vector from adjacent channel vectors by linear interpolation.
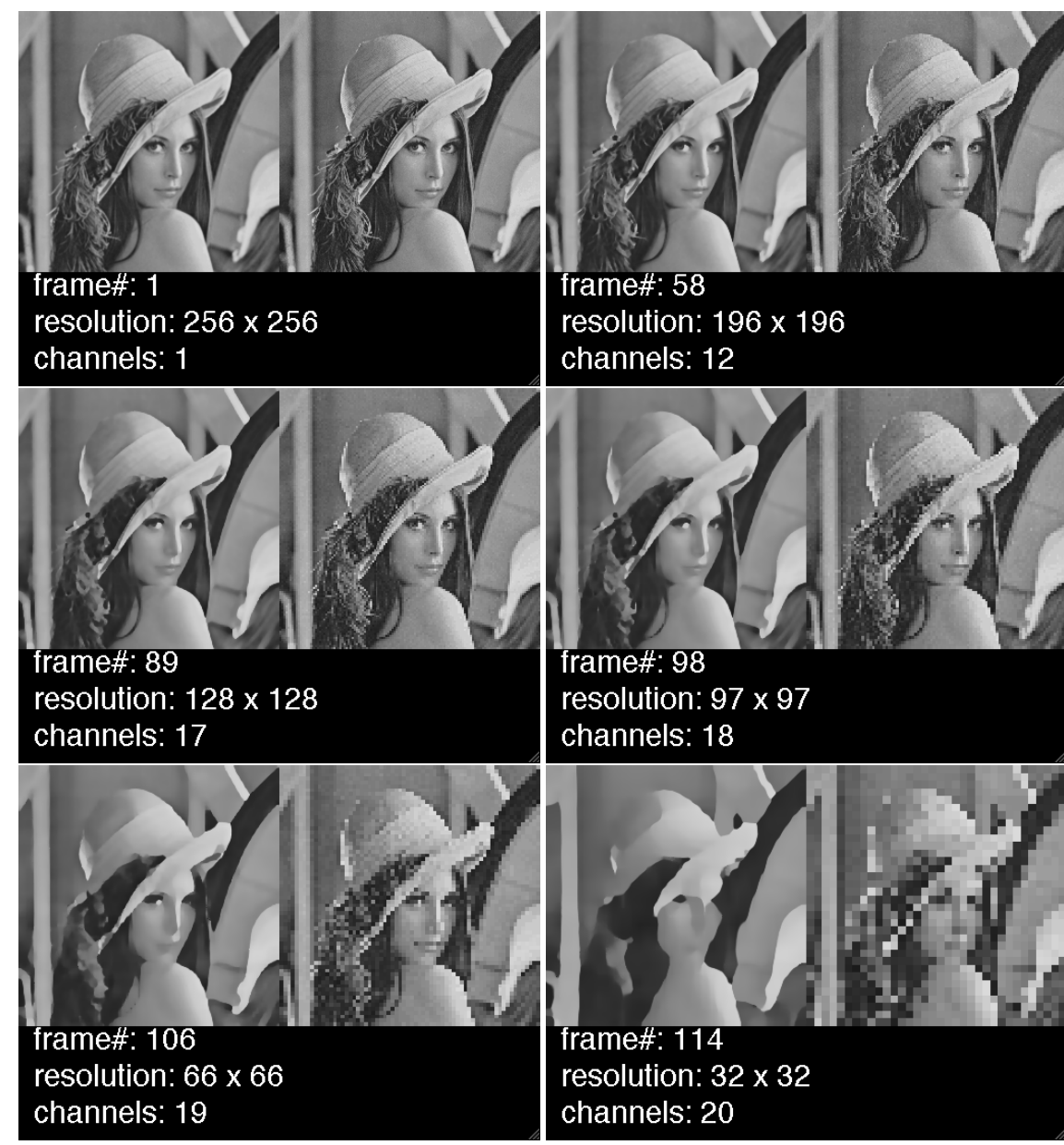

Fig. 9 Examples for CCFM-smoothing at different scales. The spatial and featural (denoted as channels) resolutions are given according to the spatio-featural uncertainty. The feature considered here is the greyscale. 


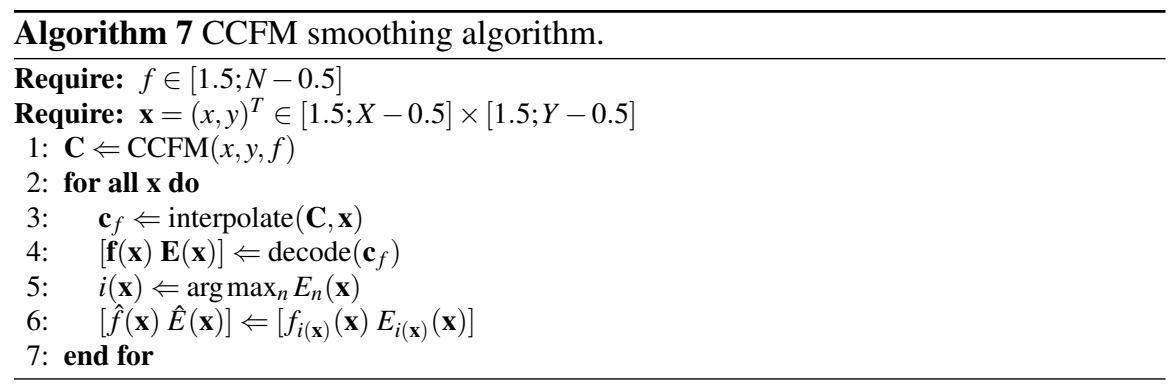

\section{Conclusion}

In this review paper, we have given a compact and concise overview over the field of channel-based filtering. Research on this topic is still in progress and more results on the efficient computation and other types of features are to be expected in the near future. Code for most of the presented work is available at the authors website.

Acknowledgements The authors would like to thank P.-E. Forssen for various discussions about the paper, in particular on alpha-synthesis.

\section{References}

1. van Almsick, M.: An a priori model of line propagation. In: L.M.J. Florack, L. Davies (eds.) Local Adaptation in Image and Signal Analysis. Springer (2009)

2. van den Boomgaard, R.: Nonlinear diffusion in computer vision. http://staff. science.uva.nl/ rein/nldiffusionweb/material.html. (Accessed 28 Jan 2008)

3. Boykov, Y., Kolmogorov, V.: An experimental comparison of min-cut/max-flow algorithms for energy minimization in vision. IEEE transactions on Pattern Analysis and Machine Intelligence 26(9), 1124-1137 (2004)

4. Burgeth, B., Pizarro, L., Didas, S., Weickert, J.: Coherence-enhancing diffusion filtering for matrix fields. In: L.M.J. Florack, L. Davies (eds.) Local Adaptation in Image and Signal Analysis. Springer (2009)

5. Burt, P.J., Adelson, E.H.: The Laplacian pyramid as a compact image code. IEEE Trans. Communications 31(4), 532-540 (1983)

6. Daubechies, I.: Orthonormal bases of compactly supported wavelets. Communications on Pure and Applied Mathematics 41(7), 909-996 (1988)

7. Duits, R., Felsberg, M., Granlund, G.H., ter Haar Romeny, B.M.: Image analysis and reconstruction using a wavelet transform constructed from a reducible representation of the Euclidean motion group. International Journal of Computer Vision 72(1), 79-102 (2007)

8. Duits, R., Florack, L.M.J., de Graaf, J., ter Haar Romeny, B.M.: On the axioms of scale space theory. Journal of Mathematical Imaging and Vision 20, 267-298 (2004)

9. Elder, J.H., Zucker, S.W.: Local scale control for edge detection and blur estimation. IEEE Trans. Pattern Analysis and Machine Intell. 20(7), 699-716 (1998) 
10. Felsberg, M.: Wiener channel smoothing: Robust Wiener filtering of images. In: DAGM 2005, LNCS, vol. 3663, pp. 468-475. Springer (2005)

11. Felsberg, M.: Extending graph-cut to continuous value domain minimization. In: Fourth Canadian Conference on Computer and Robot Vision, pp. 274-281 (2007)

12. Felsberg, M.: On the relation between anisotropic diffusion and iterated adaptive filtering. In: 30. DAGM Symposium Mustererkennung, LNCS, vol. 5096, pp. 436-445. Springer (2008)

13. Felsberg, M.: Spatio-featural scale-space. In: International Conference on Scale Space Methods and Variational Methods in Computer Vision, LNCS, vol. 5567 (2009)

14. Felsberg, M., Forssén, P.E., Scharr, H.: Channel smoothing: Efficient robust smoothing of lowlevel signal features. IEEE Transactions on Pattern Analysis and Machine Intelligence 28(2), 209-222 (2006)

15. Felsberg, M., Granlund, G.: Anisotropic channel filtering. In: Proc. 13th Scandinavian Conference on Image Analysis, LNCS 2749, pp. 755-762 (2003)

16. Felsberg, M., Granlund, G.: P-channels: Robust multivariate m-estimation of large datasets. In: International Conference on Pattern Recognition. Hong Kong (2006)

17. Felsberg, M., Kalkan, S., Krüger, N.: Continuous dimensionality characterization of image structures. Image and Vision Computing 27, 628-636 (2009)

18. Felsberg, M., Sommer, G.: The monogenic scale-space: A unifying approach to phase-based image processing in scale-space. Journal of Mathematical Imaging and Vision 21, 5-26 (2004)

19. Florack, L.M.J.: Scale space representations locally adapted to the geometry of base and target manifold. In: L.M.J. Florack, L. Davies (eds.) Local Adaptation in Image and Signal Analysis. Springer (2009)

20. Forssén, P.E.: Low and medium level vision using channel representations. Ph.D. thesis, Linköping University, Sweden (2004)

21. Forssén, P.E., Granlund, G.: Robust multi-scale extraction of blob features. In: Proceedings of the 13th Scandinavian Conference on Image Analysis, LNCS 2749, pp. 11-18 (2003)

22. Förstner, W.: Image preprocessing for feature extraction in digital intensity, color and range images. In: Proc. Int'l. Summer School on Data Analysis and the Statistical Foundation of Geomatics, LNES. Springer (1998)

23. Freeman, W.T., Adelson, E.H.: The design and use of steerable filters. IEEE Trans. Pattern Analysis and Machine Intell. 13(9), 891-906 (1991)

24. Führ, H.: Continuous diffusion wavelet transforms and scale space over Euclidean spaces and noncommutative Lie groups. In: L.M.J. Florack, L. Davies (eds.) Local Adaptation in Image and Signal Analysis. Springer (2009)

25. Granlund, G.H.: In search of a general picture processing operator. Computer Graphics and Image Processing 8, 155-173 (1978)

26. Granlund, G.H.: An associative perception-action structure using a localized space variant information representation. In: Proc. Int. Workshop on Algebraic Frames for the PerceptionAction Cycle. Springer, Heidelberg (2000)

27. Gur, Y., Pasternak, O., Sochen, N.: Fast $G L(n)$-invariant framework for tensors regularization. In: L.M.J. Florack, L. Davies (eds.) Local Adaptation in Image and Signal Analysis. Springer (2009)

28. Haindl, M.: Visual data recognition and modelling based on local Markovian models. In: L.M.J. Florack, L. Davies (eds.) Local Adaptation in Image and Signal Analysis. Springer (2009)

29. Howard, I.P., Rogers, B.J.: Binocular Vision and Stereopsis. Oxford University Press, Oxford, UK (1995)

30. Iijima, T.: Basic theory of pattern observation. In: Papers of Technical Group on Automata and Automatic Control, IECE, Japan (1959)

31. Jonsson, E.: Channel-coded feature maps for computer vision and machine learning. Ph.D. thesis, Linköping University, Sweden, SE-581 83 Linköping, Sweden (2008). Dissertation No. 1160, ISBN 978-91-7393-988-1

32. Jonsson, E., Felsberg, M.: Reconstruction of probability density functions from channel representations. In: Proc. 14th Scandinavian Conference on Image Analysis (2005) 
33. Jonsson, E., Felsberg, M.: Accurate interpolation in appearance-based pose estimation. In: Proc. 15th Scandinavian Conference on Image Analysis, LNCS, vol. 4522, pp. 1-10 (2007)

34. Jonsson, E., Felsberg, M.: Efficient computation of channel-coded feature maps through piecewise polynomials. Image and Vision Computing http://dx.doi.org/10.1016/j. imavis .2008.11.002 (2009)

35. Knutsson, H., Westin, C.F.: Normalized convolution: Technique for filtering incomplete and uncertain data. In: Proceedings of the 8th Scandinavian Conference on Image Analysis, vol. II, pp. 997-1006 (1993)

36. Knutsson, H., Wilson, R., Granlund, G.H.: Anisotropic non-stationary image estimation and its applications: Part I - restoration of noisy images. IEEE Trans. on Communications COM31(3), 388-397 (1983)

37. Koenderink, J.J.: The structure of images. Biological Cybernetics 50, 363-370 (1984)

38. Koenderink, J.J., van Doorn, A.J.: Image processing done right. In: Proceedings European Conference on Computer Vision, pp. 158-172 (2002)

39. Lindeberg, T.: Scale-Space Theory in Computer Vision. The Kluwer International Series in Engineering and Computer Science. Kluwer Academic Publishers, Boston (1994)

40. Loubes, J.M., Rochet, P.: Regularization with approximated $L^{2}$ maximum entropy method. In: L.M.J. Florack, L. Davies (eds.) Local Adaptation in Image and Signal Analysis. Springer (2009)

41. Lüdtke, N.L., Wilson, R.C., Hancock, E.R.: Probabilistic population coding of multiple edge orientation. In: Proceedings of IEEE International Conference on Image Processing, vol. II, pp. 865-868 (2002)

42. Mallat, S.G.: A theory for multiresolution signal decomposition: the wavelet representation. IEEE Transactions on Pattern Analysis and Machine Intelligence 11, 674-693 (1989)

43. Paris, S., Durand, F.: A fast approximation of the bilateral filter using a signal processing approach. In: European Conference on Computer Vision (2006)

44. Perona, P., Malik, J.: Scale-space and edge detection using anisotropic diffusion. IEEE Trans. Pattern Analysis and Machine Intelligence 12(7), 629-639 (1990)

45. Polzehl, J., Tabelow, K.: Structural adaptive smoothing: Principles and applications. In: L.M.J. Florack, L. Davies (eds.) Local Adaptation in Image and Signal Analysis. Springer (2009)

46. Portilla, J., Strela, V., Wainwright, J., Simoncelli, E.P.: Image denoising using scale mixtures of Gaussians in the wavelet domain. IEEE Trans. Image Processing 12(11), 1338-1351 (2003)

47. Pouget, A., Dayan, P., Zemel, R.: Information processing with population codes. Nature Reviews - Neuroscience 1, 125-132 (2000)

48. Rozenholc, Y., Reiss, M., Cuenod, C.A.: Preserving time structures while denoising dynamical image. Application to dynamic contrast enhanced-CT. In: L.M.J. Florack, L. Davies (eds.) Local Adaptation in Image and Signal Analysis. Springer (2009)

49. Scharr, H., Felsberg, M., Forssén, P.E.: Noise adaptive channel smoothing of low-dose images. In: CVPR Workshop: Computer Vision for the Nano-Scale (2003)

50. Scharr, H., Krajsek, K.: A short introduction to diffusion-like methods. In: L.M.J. Florack, L. Davies (eds.) Local Adaptation in Image and Signal Analysis. Springer (2009)

51. Therrien, C.W.: Decision, estimation, and classification: an introduction into pattern recognition and related topics. John Wiley \& Sons, Inc. (1989)

52. Weickert, J.: Theoretical foundations of anisotropic diffusion in image processing. Computing, Suppl. 11, 221-236 (1996)

53. Weickert, J., Ishikawa, S., Imiya, A.: Linear scale-space has first been proposed in Japan. Mathematical Imaging and Vision 10, 237-252 (1999)

54. Weickert, J., Scharr, H.: A scheme for coherence-enhancing diffusion filtering with optimized rotation invariance. Journal of Visual Communication and Image Representation, Special Issue On Partial Differential Equations In Image Processing, Computer Vision, And Computer Graphics pp. 103-118 (2002)

55. Witkin, A.P.: Scale-space filtering. In: Proc. 8th Int. Joint Conf. Art. Intell., pp. 1019-1022 (1983)

56. Zemel, R.S., Dayan, P., Pouget, A.: Probabilistic interpretation of population codes. Neural Computation 10(2), 403-430 (1998) 